\title{
Study of $\alpha$ B-Crystallin Expression in Gerbil BCAO Model of Transient Global Cerebral Ischemia
}

\author{
Ting Li, Xiaoye Mo, Zheng Jiang, Wenfang He, Wei Lu, He Zhang, Jie Zhang, \\ Liuwang Zeng, Binbin Yang, Han Xiao, and Zhiping Hu \\ Department of Neurology, The Second Xiangya Hospital, Central South University, Changsha 410011, China \\ Correspondence should be addressed to Zhiping Hu, huzhiping2007@yahoo.com.cn
}

Received 31 July 2012; Revised 13 September 2012; Accepted 14 September 2012

Academic Editor: Daniela Giustarini

Copyright $\odot 2012$ Ting Li et al. This is an open access article distributed under the Creative Commons Attribution License, which permits unrestricted use, distribution, and reproduction in any medium, provided the original work is properly cited.

\begin{abstract}
$\alpha \mathrm{B}$-crystallin ( $\alpha$-BC), the fifth member of mammalian small heat shock protein family (HspB5), is known to be expressed in many tissues and has a distinctive interaction with cytoskeleton components. In this study, we investigated that $\alpha$-BC and microtubuleassociated protein-2 (MAP-2), a neuron-specific cytoskeleton protein, were coexpressed in neurons of Gerbil cortex, while in subcortex Gerbil brains, we found that several MAP-2-negative glia cells also express $\alpha$-BC. When subjected to 10-minute bilateral carotid artery occlusion (BCAO), an increment was observed in $\alpha$-BC-positive cells after 6 -hour reperfusion and peaked at around 7 days after. In the same circumstances, the number and the staining concentration of MAP-2 positive neurons significantly decreased immediately after 6-hour reperfusion, followed by a slow recovery, which is consistent with the increase of $\alpha$-BC. Our results suggested that $\alpha$-BC plays an important role in brain ischemia, providing the early protection of neurons by giving intracellular supports through the maintenance of cytoskeleton and extracellular supports through the protection of glia cells.
\end{abstract}

\section{Introduction}

$\alpha \mathrm{B}$-crystallin/HspB5 ( $\alpha$-BC) is one member of the mammalian small heat shock protein family which consists of ten known members from HspB1 to B10. Though isolated from the crystallin lens, it has been described in a broad number of tissues and organs including the brain, skeletal muscle, heart, and kidney, and it is shown to implicate in many diseases, such as multiple sclerosis (MS), Guillain-Barre syndrome (GBS), Alexander disease, epilepsy, Down syndrome, familial amyotrophic lateral sclerosis (FALS), familial amyloidotic polyneuropathy and chronic inflammatory demyelinating polyneuropathy [1-8].

It has been reported that $\alpha$-BC functions as molecular chaperones by binding with denatured protein under stress in a reversible equilibrium state. In addition, the specific interaction between $\alpha$-BC and cytoskeletal structures in cardiac and skeletal myocytes has been proved, and the interaction is enhanced after stress, which contributes to increased stress tolerance [9-12]. The increased expression of $\alpha$-BC in cerebral arteriovenous malformations (AVMs) is also associated with maintenance of the intermediate fibre (IF) network, which increases wall tension caused by dilating vessels and the hemodynamic stress surrounding [13], another research found out that $\alpha$-BC significantly suppressed the ADP-induced secretions of both platelet-derived growth factor (PDGF) and serotonin by inhibition of HSP27 phosphorylation via p44/p42 MAPK and p38 MAPK [14]. According to its antihemodynamic stress and antiplatelet aggregation effects, we speculate that it plays an important role in ischemic vascular diseases. In addition, research data identified $\alpha$-BC as downstream effector of calcineurininduced protection against apoptosis, [15] and it is sufficient to inhibit apoptosis by interacting with apoptosis-associated factors such as Bax, Bcl-XS, p53, caspase-3 and its activators [16-20].

Moreover, $\alpha$-BC is shown to exhibit a potential role in neurological diseases involving protection of atrophy in myopathy, prevention of abnormal protein aggregation in neurodegenerative diseases, and involvement in immune responses of nervous system [21]. However, it is a relative newcomer in brain ischemia diseases. 
Cytoskeleton is composed of microtubule, microfilament, and intermediate filament, and it is involved in maintaining cell structure and participating in cellular kinesis, cleavage, and nutrient substance transportation, and it also plays a crucial role in signal transduction. Both heat stress and ischemia can cause extensive damage to the cytoskeleton, and early change in cytoskeleton is a sensitive index of affected cells [22]. Microtubule-associated protein2 (MAP-2) is a neuron-restricted cytoskeleton protein [23] and serves as a substrate for most of protein kinases and phosphatases present in neurons. Moreover, MAP-2 may perform many functions including the nucleation and stabilization of microtubules and the regulation of organelle transport within axons and dendrites in response to different extracellular signals $[23,24]$. Fujita et al. reveal that $\alpha$ BC binds microtubules (MTs) via microtubule-associated proteins (MAPs) and gives the MTs resistance to calciuminduced disassembly [25].

In this study, we showed that $\alpha$-BC was coexpressed with MAP-2 in cortex neurons and also in a group of MAP-2-negative glia cells in subcortex Gerbil brains by double immunofluorescence. Staining characteristic of $\alpha$-BC in these cells is the concentrated cytoplasm staining around the nucleus and extending to dendrites. While subjected to 10-minute bilateral carotid artery occlusion (BCAO), the expression of $\alpha$-BC was gradually upregulated from 6 hours to 7 days after reperfusion. However, MAP-2-positive neurons showed significant decrease in number and staining intensity as early as 6 hours after reperfusion and then recovered gradually, which is accompanied by the increase of $\alpha$-BC. We speculate that $\alpha$-BC plays an important role in brain ischemia.

\section{Materials and Methods}

2.1. Animals and Surgery. All animal experiments were conducted in accordance with National Institutes of Health and institutional guidelines. Male Mongolian Gerbils (Hangzhou, Zhejiang, China) weighing 80-90 g were housed in standard temperature $\left(22 \pm 1^{\circ} \mathrm{C}\right)$ and light-controlled (light on 07:00-20:00) environment with ad libitum access to food and water. The animals were randomly divided into two groups. They were sham and BCAO groups, and each group was divided into four time points (6 hours, 24 hours, 72 hours, and 7 days after surgery). In addition, there was a group of Gerbils without any treatment. There were eight animals in each group. Before surgery, each animal was anesthetized by peritoneal injection with $3 \%$ pentobarbital $(30 \mathrm{mg} / \mathrm{kg})$. Bilateral carotid artery occlusion (BCAO) was carried out as described by $\mathrm{Hu}$ et al. [26] Occlusion was maintained for 10 minutes, and this was followed by reperfusion for $6 \mathrm{~h}, 24 \mathrm{~h}, 72 \mathrm{~h}$, and 7 days. In sham-operated animals, the arteries were exposed but not occluded. The wound was then well sutured, and the animals were allowed to recover. The body temperature was maintained at $36-37^{\circ} \mathrm{C}$ during the surgery with a heating pad. The success of this ischemia/reperfusion model was further proved and evaluated by $\mathrm{HE}$ staining of Gerbil cerebral cortex with the following indications: intracellular or interstitial edema, inflammatory infiltration, and gliosis.

2.2. Immunohistochemical Methods. At the time points, the animals were perfused transcardially with $30 \mathrm{~mL}$ of $0.9 \%$ saline followed by $100 \mathrm{~mL}$ of $4 \%$ paraformaldehyde solution. The brains were removed and placed in $10 \%$ formalin for less than $24 \mathrm{~h}$, processed, and embedded in paraffin wax. Coronal sections $(5 \mu \mathrm{m})$ of brain were taken using a microtome. Immunohistochemical staining was performed on paraffin-embedded coronal sections $(5 \mu \mathrm{m})$. Sections were deparaffinized and rehydrated. Sections were then incubated in $0.3 \%$ hydrogen peroxide $\left(\mathrm{H}_{2} \mathrm{O}_{2}\right)$ for $5 \mathrm{~min}$ to block endogenous peroxidase activity, rinsed, incubated in pepsin/ $\mathrm{HCl}$ for $30 \mathrm{~min}$, rinsed in water, and then rinsed in PBS (PH 7.2-7.6, 0.02 M). Normal goat serum (1.5\% in PBS) was used as a preblock for 20 minutes after which the sections were incubated with primary antibody $\alpha$-BC (Santa Cruz, FL 175, USA) $1: 150$ and primary antibody MAP-2 (Boster, BA0056), respectively, at $37^{\circ} \mathrm{C}$ in a humidified immunostaining chamber for 1 hour. Sections were then rinsed in PBS (4 $\times 10 \mathrm{~min}$ ) and incubated in biotinylated secondary antibody for $20 \mathrm{~min}$ at room temperature, after which they were again washed in PBS $(3 \times 10 \mathrm{~min})$. Subsequently, sections were incubated in avidin-conjugated horseradish peroxidase for $20 \mathrm{~min}$ at room temperature and washed in PBS (4 $\times 5 \mathrm{~min}$ ). Finally, sections were visualized by using $0.05 \%$ diaminobenzidine and $0.02 \% \mathrm{H}_{2} \mathrm{O}_{2}$ until color developed and then washed in tap water, dehydrated, coverslipped using DPX mountant, and examined under a light microscope. Expression index: under high magnification $(10 \times 40)$ or oil microscope $(10 \times 100)$, count the numbers of positive cells with cytoplasm brown particles per 1000 cells in randomly selected areas of each slice.

2.3. Double Immunofluorescence. Immunofluorescence double labeling was also performed on paraffin-embedded coronal sections $(5 \mu \mathrm{m})$. Sections were deparaffinized and rehydrated in a normal way. Primary antibodies were diluted at $1: 100$ for anti- $\alpha$-BC (Santa Cruz, FL-175, USA) and at 1:150 for anti-MAP-2 (Boster, BA0056) antibodies. For double immunostaining, 1:200 TRITC-labeled anti-rabbit IgG (Boster) was used as the secondary antibody for anti- $\alpha$ $\mathrm{BC}$ antibodies. After washing with 0.1 M PH7.4 PBS, sections were incubated with $1: 200$ FITC-conjugated anti-mouse IgG (Boster) for anti-MAP-2-antibodies in PBS for $30 \mathrm{~min}$ at room temperature. Sections were observed by fluorescence microscope under $546 \mathrm{~nm}$ and $490 \mathrm{~nm}$ wavelength excitation light, TRITC-labeled a-BC-positive cells presented red fluorescence, and FITC-labeled MAP-2-positive cells presented green fluorescence.

\section{Results}

3.1. Expression of $\alpha B$-Crystallin in Cortex Neurons and a Group of Glia Cells after Ischemia/Reperfusion (I/R) Injury. In the brain slices of Gerbil BCAO/reperfusion model, immuno-fluorescence staining of cerebral cortex showed 
MAP-2-labeled neurons with green fluorescence and $\alpha$-BCpositive cells with red fluorescence (Figure 1); we found that the fluorescence of $\alpha$-BC largely overlapped with that

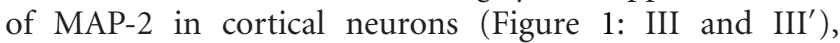
suggesting the coexpression and colocation of both proteins in neurons after ischemia/reperfusion (I/R) injury. Thus, $\alpha$-BC may interact with MAP-2 to further protect the microtubules (MTs) in cortical neurons after I/R injury, while in subcortical brain tissue slices, we also found out a group of MAP-2-negative nonneuronal glia cells which were $\alpha$-BC positive (Figure 1: I, I' and II, $\mathrm{II}^{\prime}$ ) (white arrow head), suggesting that $\alpha$-BC was also expressed in a group of glia cells.

\subsection{Annular Cytoplasmic Expression of $\alpha B$-Crystallin in Glia} Cells. The nuclei of $\alpha$-BC-positive glia cells were surrounded by densely stained annular cytoplasm, and the staining is further extended into proximal segments of cytodendrites (Figure 1: IV, V and VI, VII). Expression of $\alpha$-BC-in neurons was relatively stable during $I / R$ injury. While, a significant increment in the staining concentration and the number of typical $\alpha$-BC positive glia cells was detected after $\mathrm{BCAO} /$ reperfusion (Figures $2(\mathrm{a})-2(\mathrm{~d})$ ), the upregulated expression appeared after 6-hour reperfusion and sustained for 7 days. The expression of MAP-2 was prominently in neurons and less detected in glia cells, which was further confirmed by double immunofluorescent staining (Figure 1: I, I' and II, II').

3.3. The Expression of MAP-2 and $\alpha-B C$ in the Normal and Ischemia/Reperfusion Gerbil Brains. Previous researches suggest that $\alpha$-BC may interact with cytoskeleton components to give the first line of defense during ischemia/reperfusion injury [10]. We use DAB staining to observe the expression of $\alpha$-BC and MAP-2 in Gerbil brain slices. Expression index is calculated by counting the numbers of positive cells with cytoplasm brown particles per 1000 cells in randomly selected areas of each slice under high magnification $(10 \times$ $40)$. Results indicated that $\alpha$-BC was induced in the early $\mathrm{BCAO/reperfusion} \mathrm{brain} \mathrm{as} \mathrm{soon} \mathrm{as} 6$ hours after reperfusion, while MAP-2 was significantly reduced immediately after $\mathrm{BCAO} /$ reperfusion to almost $20 \%$ of normal level followed by a slow recovery (Figures $2(\mathrm{a})-2(\mathrm{~h})$; Figure 3 ). The expression index trendgram (Figure 3 ) of $\alpha$-BC and MAP2 shows that the recovered expression of MAP-2 following sharp decrease after I/R injury is accompanied with the upregulation of $\alpha$-BC. The correlation between MAP- 2 and $\alpha$-BC during brain I/R ischemia suggests that the molecular chaperone $\alpha$-BC may protect neurons by preventing the degradation of denatured MAP-2.

\section{Discussion}

$\alpha$-BC is constitutively expressed in the central nervous system (CNS), and its level of expression in the normal central nervous system is low and appears to be centralized to glia cells, both astrocytes and oligodendrocytes [27]. Many neurodegenerative disorders including Alzheimer's Disease, and Parkinson's disease, amyotrophic lateral sclerosis (ALS) are characterized by conformational changes in proteins that result in misfolding, aggregation, and intra- or extraneuronal accumulation of amyloid fibrils. $\alpha$-BC, as a molecular chaperone, provides a first line of defense against misfolded, aggregation-prone proteins in central nervous system (CNS) [28], while, Narayanan et al. [29] demonstrated that in the presence of $\alpha-\mathrm{BC}, \mathrm{A} \beta$ became efficiently oxidized with increased neurotoxicity and the inability to form fibrillar structures. Results are paradoxical, and the exactly role of $\alpha$ BC still remains a matter to be concerned about.

Multiple sclerosis (MS) is a chronic neurodegenerative disease of the CNS with extensive demyelination, loss of oligodendrocytes, and axonal degeneration. In MS, T-cell response to $\alpha$-BC is much stronger than all other components of myelin in MS and is considered to be a potential autoantigen [30]. Ousman et al. suggested that the immune response against $\alpha$-BC in multiple sclerosis would exacerbate inflammation and demyelination, which can be countered by recombinant CRYAB for therapy of ongoing disease [21]. However, research by Rothbard et al. demonstrated that the putative anti-HspB5/ $\alpha$-BC Abs from 23 MS patients crossreact with 7 other members of the human small Hsp family and were equally present in normal plasma. These results refute much of the serological data used to assign $\alpha$-BC as an autoantigen [8].

Alexander disease $(\mathrm{AxD})$ is a primary disorder of astrocytes caused by dominant mutations in the gene for glia fibrillary acidic protein (GFAP). In AxD mouse models, loss of CRYAB results in increased mortality, whereas elevation of CRYAB rescues animals from terminal seizures by regulation of GFAP assembly and reduction in CNS stress response [1].

In our $\mathrm{BCAO/reperfusion} \mathrm{Gerbil} \mathrm{model,} \mathrm{the} \alpha$-BC immune-reactivity is detected in the cytoplasm of both neurons and a group of glia cells, which is in agreement with previous research by Piao et al. [31]. In their research of rat brain after middle cerebral artery occlusion (MCAO), they demonstrated one group of glia cells which only expressed $\alpha$ $\mathrm{BC}$ and were $\mathrm{p} 38 \beta \mathrm{MAPK}$ or MAPKAPK-2 negative; a further study revealed that those cells were oligodendrocytes. Moreover, in subcortical Gerbil brains, $\alpha$-BC immune reactivity was upregulated gradually from 6 hours after reperfusion to peak at around 7 days after. An increment was observed in the number and the staining concentration of $\alpha$-BCpositive cells. Staining feature of these glia cells is described as annular cytoplasmic dense staining, which is distributed around the nucleus and extended into proximal segments of cytodendrites.

Recently, several studies have investigated the close interaction between $\alpha \mathrm{B}$-crystallin and cytoskeletal components in nonneural cells, such as cardiac muscle cells, skeletal muscle cells, vascular endothelial cells, collagenoblasts, and osteoblasts (reviewed by $\mathrm{Hu}$ and $\mathrm{Li}$ [32]). In unstimulated cardiac myocytes, biochemical studies indicate that $\alpha$-BC associates with cytoskeletal components and is highly soluble and remains in the cytosolic fraction; heat or ischemia triggers rapid translocation of $\alpha$-BC into the insoluble cytoskeletal/nuclear fractions [33] with enhanced interaction with cytoskeletal components [9-12]. Thus, it is important 

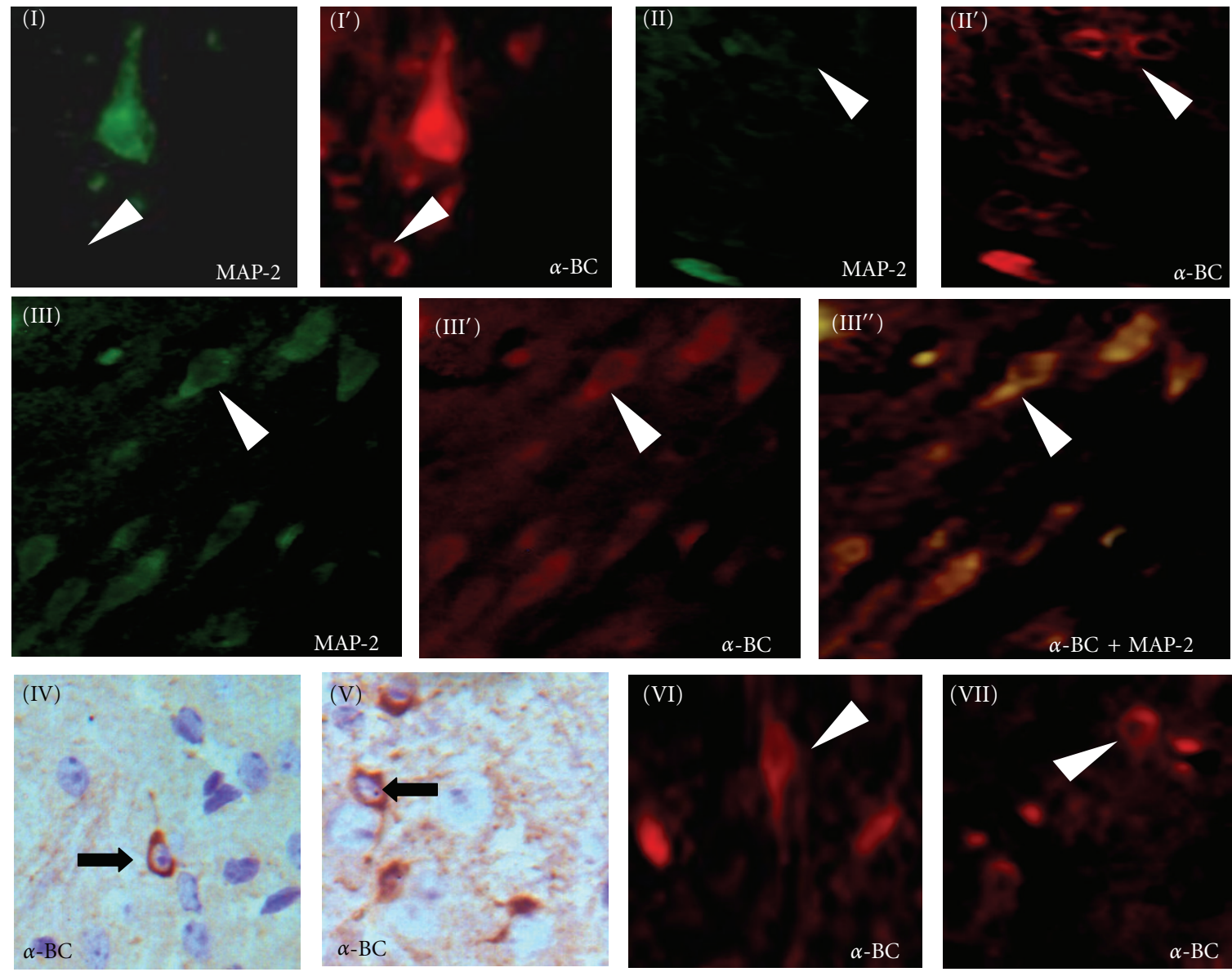

FIGURE 1: Induction of MAP-2 (in green fluorescence) and $\alpha \mathrm{B}$-crystallin ( $\alpha$-BC) (in red fluorescence) in subcortical tissue sections and parietal cortex brain slices the in 7th day of reperfusion after transient global cerebral ischemia in Gerbil model of BCAO. $\alpha$-BC-positive but MAP-2-negative nonneuronal glia cells (I, I' and II, II') (white arrow head) in subcortical tissue sections; co-expression of MAP-2 with $\alpha$-BC in neurons of parietal cortex $\left(\mathrm{III}^{\prime \prime}\right)$ (in yellow fluorescence); DAB $(\times 40)$ and immunofluorescence staining $(\times 40)$ of normal subcortical brain sections shows that the nucleus is surrounded by densely stained cytoplasm, and the staining is further extended into proximal segments of cytodendrites in $\alpha$-BC-positive cells (IV, V and VI, VII). (Black arrow and white arrow head resp.).

for $\alpha$-BC to confer early protection of ischemic cardiac. In addition, recent researches indicate that $\alpha$-BC can suppress the aggregation of platelets [14] and provide protection under hemodynamic stress [13] which prompts its potential significance in vascular diseases.

MAP-2 is a cytoskeleton component protein found mainly in dendrites and soma of neuronal cells in most regions of central nervous system, and it has been used extensively as a very sensitive and specific marker for neuronal differentiation during the last two decades [34]. It can modulate the assembling of tubulin/microtubule which commonly allows the cell to respond mechanically to the environment. Loss of MAP-2 by degradation of calpain I in both the striatum and hippocampus after ischemia in the Gerbil forebrain was found to occur earlier than spectrin degradation by Western blot analysis [35]. Those results suggest that dendritic loss of MAP-2 immunoreactivity after ischemia might participate in the initial phase of neuronal cytoskeleton dysfunction and might become a first sign of neurodegeneration. Researches of permanent middle cerebral artery occlusion (MCAO) by Dawson and Hallenbeck [36] also elicited a rapid reduction in MAP-2 immunostaining that was visible $1 \mathrm{~h}$ after MCAO, indicating that MAP-2 could be used in acute brain injury following focal ischemia as a sensitive and quantifiable indicator. Here, we used MAP- 2 as a neuron marker and found that $\alpha$-BC and MAP-2 were colocated in neurons of cerebral cortex in Gerbil BCAO/reperfusion model by double immunofluorescence staining; in addition, a group of MAP-2-negative glia cells also strongly expressed $\alpha$-BC in subcortical brain, suggesting the broad expression of $\alpha$-BC in the CNS. When confronted with ischemia/reperfusion injury, the MAP-2positive neurons were significantly reduced immediately after reperfusion to almost $20 \%$ of normal level followed by a slow recovery, which was consistent with previous researches. The recovered expression of MAP-2 following sharp decrease was accompanied with sustained upregulation of $\alpha$-BC since the third day after reperfusion. The correlation between MAP-2 and $\alpha$-BC in cortex neurons during brain I/R ischemia suggests that the molecular chaperone $\alpha$-BC may 


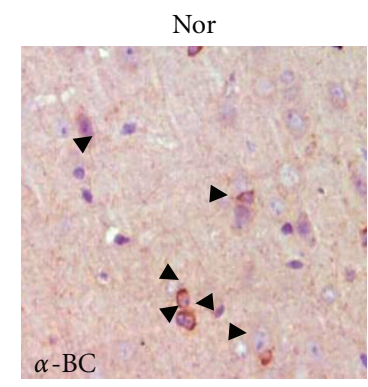

(a)

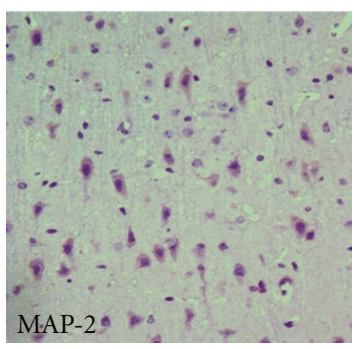

(e)

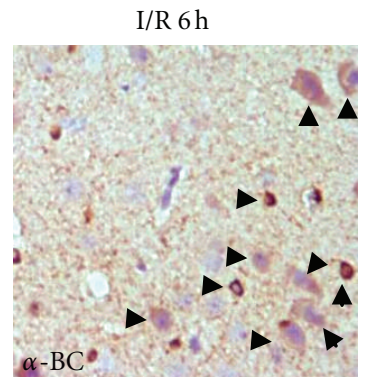

(b)

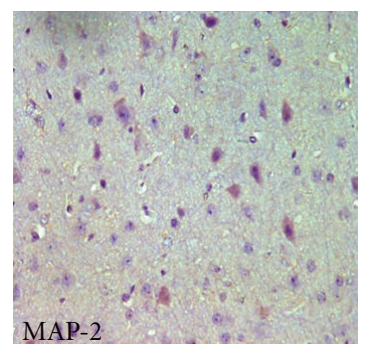

(f)

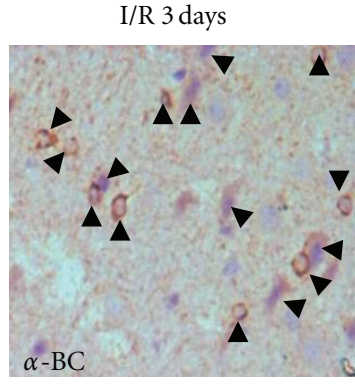

(c)

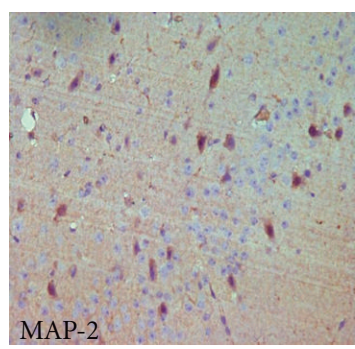

(g)

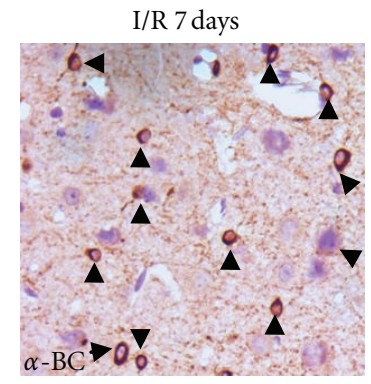

(d)

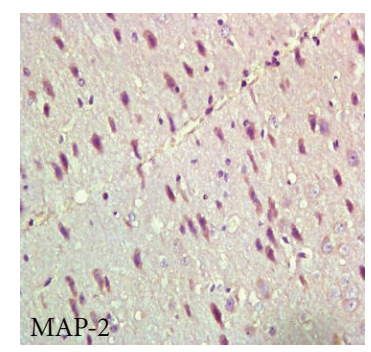

(h)

FIGURE 2: Immunohistochemical findings $(\mathrm{DAB} \times 20)$ of $\alpha$-BC or MAP-2 in Gerbil brain sections of normal $(\mathrm{A} / \mathrm{E})$ and ischemia/reperfusion (I/R) 6 hours $(\mathrm{B} / \mathrm{F}), 3$ days $(\mathrm{C} / \mathrm{G})$, and 7 days $(\mathrm{D} / \mathrm{H})$ after occlusion. An increment in the number of $\alpha$-BC-positive cells (black arrow head) was detected 6 hours after reperfusion and lasted until the 7th day after reperfusion; an immediate reduction of MAP-2-positive neurons in 6 hours after reperfusion was detected and began to recover after 3 days post-reperfusion, on the 7th day the number of MAP-2-positive neurons was almost normal.

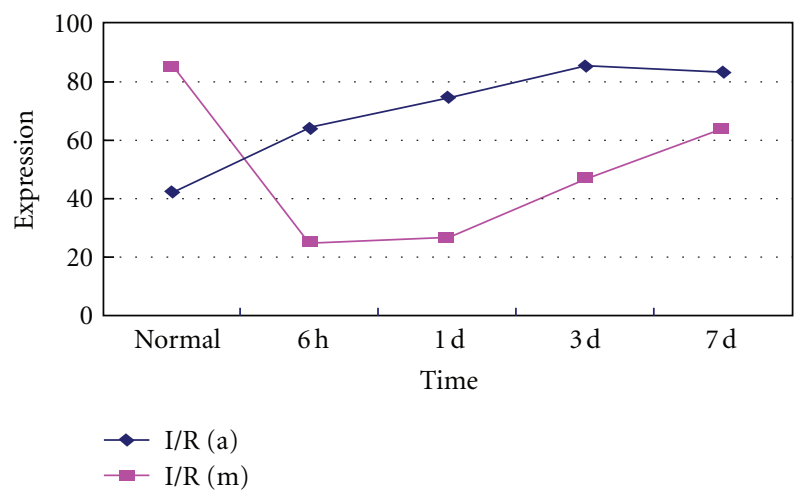

Figure 3: Trendgram of $\alpha$-BC (blue line) and MAP-2 (red line) in normal and ischemia/reperfusion Gerbil brains: an increment in the expression index of $\alpha$-BC-positive cells and a sharp decrement and slow recovery expression index of MAP-2-positive cells in normal and BCAO in the 6 hours, 1 day, 3 days, and 7 days after reperfusion of Gerbil brains were detected and showed above.

protect neurons by preventing the degradation of MAP-2 via enhanced interaction.

All complex nervous systems consist of two main cell types, neurons and glia cells. The evolution of increasing complexity in the nervous system is accompanied by a steady rise in glia cell number. Oligodendrocytes in the central nervous system (CNS) produce the myelin sheath of neurons by wrapping of its plasma membrane extensions around the axon, and compacting with the stacked membrane bilayers. They also modify the axonal cytoskeleton and the rate of vesicular transport along the axons [37-39]. The reciprocal communication between neurons and oligodendrocytes is important for the development of the nervous system. Furthermore, there is mounting evidence that neuron-glia communication has a role in the pathogenesis of many neurological diseases.

During brain ischemia, neurons and glia cells upregulated the expression of $\alpha$-BC against the injuries caused by free radicals, calcium overload, releasing of proteinase, and so on probably through at the least four aspects below (reviewed by $\mathrm{Hu}$ and $\mathrm{Li}$ [32]): (1) translocating to myofilament and nuclear structures where the chaperone functions confer cytoskeleton stabilization; (2) associating with apoptosis regulators such as Bax, Bcl-XS, p53, caspase3 , and its activators conferring apoptosis; (3) interacting with other cellular proteins, especially those denatured proteins and keeping them in a soluble state waiting for refolding; (4) translocating to mitochondria, where it is phosphorylated and contributes to modulating mitochondrial damage [40]. In addition, elevated $\alpha$-BC expression of glia cells may lead to the increase of the survival rate of glia cells and their strengthened communication with neurons through axons during ischemia/reperfusion injury.

According to our research on ischemia/reperfusion animal model, $\alpha$-BC probably acts as a neuroprotective 
molecule providing the early protection of neurons by giving intracellular supports through the maintenance of cytoskeleton and extracellular supports through the protection of glia cells, and its anti-inflammatory functions during stroke need to be further explored for the increased expression in multiple glia cells. Understanding of the exact interaction between $\alpha$-BC and cytoskeleton in nerve cells and further calcification of $\alpha$-BC-specific subgroup glia cells will not only provide new exciting insights into the mechanisms of the cerebral ischemia/reperfusion injury but also help us to find new treatment strategies for stroke and uncover the pathogenesis of many other neurological diseases.

\section{Acknowledgments}

This paper and related research has been supported by Frontier Research Plan of Central South University (2010, Grant 2177-721500065); Ministry of Education Foundation (Grant 20070533060); National Natural Science Foundation (Grant 81171239), China.

\section{References}

[1] T. L. Hagemann, W. C. Boelens, E. F. Wawrousek, and A. Messing, "Suppression of GFAP toxicity by $\alpha \mathrm{B}$-crystallin in mouse models of Alexander disease," Human Molecular Genetics, vol. 18, no. 7, pp. 1190-1199, 2009.

[2] S. Palminiello, K. Jarzabek, K. Kaur et al., "Upregulation of phosphorylated $\alpha \mathrm{B}$-crystallin in the brain of children and young adults with Down syndrome," Brain Research, vol. 1268, pp. 162-173, 2009.

[3] H. Hegen, J. Wanschitz, R. Ehling et al., "Anti- $\alpha$ B-crystallin immunoreactivity in Guillain-Barré syndrome and chronic inflammatory demyelinating polyneuropathy," Journal of the Peripheral Nervous System, vol. 15, no. 2, pp. 150-152, 2010.

[4] C. M. Karch and D. R. Borchelt, "An examination of $\alpha$ bcrystallin as a modifier of SOD1 aggregate pathology and toxicity in models of familial amyotrophic lateral sclerosis," Journal of Neurochemistry, vol. 113, no. 5, pp. 1092-1100, 2010.

[5] J. Magalhães, S. D. Santos, and M. J. Saraiva, " $\alpha$ B-crystallin (HspB5) in familial amyloidotic polyneuropathy," International Journal of Experimental Pathology, vol. 91, no. 6, pp. 515-521, 2010.

[6] Y. K. Hayashi, "Myofibrillar myopathy," Brain Nerve, vol. 63, no. 11, pp. 1179-1188, 2011.

[7] J. Ojha, R. V. Karmegam, J. Gunasingh Masilamoni, A. V. Terry, and A. G. Cashikar, "Behavioral defects in chaperonedeficient Alzheimer's disease model mice," PLoS One, vol. 6, no. 2, Article ID e16550, 2011.

[8] J. B. Rothbard, X. Zhao, O. Sharpe et al., "Chaperone activity of $\alpha \mathrm{B}$-crystallin is responsible for its incorrect assignment as an autoantigen in multiple sclerosis," Journal of Immunology, vol. 186, no. 7, pp. 4263-4268, 2011.

[9] K. Djabali, B. de Nechaud, F. Landon, and M. M. Portier, “ $\alpha$ Bcrystallin interacts with intermediate filaments in response to stress," Journal of Cell Science, vol. 110, no. 21, pp. 2759-2769, 1997.

[10] N. Mercatelli, I. Dimauro, S. A. Ciafré, M. G. Farace, and D. Caporossi, " $\alpha \mathrm{B}$-crystallin is involved in oxidative stress protection determined by VEGF in skeletal myoblasts," Free Radical Biology and Medicine, vol. 49, no. 3, pp. 374-382, 2010.
[11] F. A. J. M. van de Klundert, M. L. J. Gijsen, P. R. L. A. van den Ijssel, L. H. E. H. Snoeckx, and W. W. de Jong, " $\alpha$ B-crystallin and hsp25 in neonatal cardiac cells-differences in cellular localization under stress conditions," European Journal of Cell Biology, vol. 75, no. 1, pp. 38-45, 1998.

[12] P. Verschuure, Y. Croes, P. R. L. A. van den IJssel, R. A. Quinlan, W. W. de Jong, and W. C. Boelens, "Translocation of small heat shock proteins to the actin cytoskeleton upon proteasomal inhibition," Journal of Molecular and Cellular Cardiology, vol. 34, no. 2, pp. 117-128, 2002.

[13] Y. Ha, T. S. Kim, D. H. Yoon, Y. E. Cho, S. G. Huh, and K. C. Lee, "Reinduced expression of developmental proteins (nestin, small heat shock protein) in and around cerebral arteriovenous malformations," Clinical Neuropathology, vol. 22, no. 5, pp. 252-261, 2003.

[14] Y. Enomoto, S. Adachi, R. Matsushima-Nishiwaki et al., " $\alpha \mathrm{B}$-crystallin extracellularly suppresses ADP-induced granule secretion from human platelets," FEBS Letters, vol. 583, no. 15, pp. 2464-2468, 2009.

[15] N. Bousette, S. Chugh, V. Fong et al., "Constitutively active calcineurin induces cardiac endoplasmic reticulum stress and protects against apoptosis that is mediated by $\alpha$-crystallin-B," Proceedings of the National Academy of Sciences of the United States of America, vol. 107, no. 43, pp. 18481-18486, 2010.

[16] J. H. Shin, S. W. Kim, C. M. Lim, J. Y. Jeong, C. S. Piao, and J. K. Lee, " $\alpha \mathrm{B}$-crystallin suppresses oxidative stress-induced astrocyte apoptosis by inhibiting caspase-3 activation," Neuroscience Research, vol. 64, no. 4, pp. 355-361, 2009.

[17] G. Watanabe, S. Kato, H. Nakata, T. Ishida, N. Ohuchi, and C. Ishioka, " $\alpha \mathrm{B}$-crystallin: a novel p53-target gene required for p53-dependent apoptosis," Cancer Science, vol. 100, no. 12, pp. 2368-2375, 2009.

[18] J. H. Shin, J. Y. Jeong, Y. Jin, I. D. Kim, and J. K. Lee, "P38 $\beta$ MAPK affords cytoprotection against oxidative stress-induced astrocyte apoptosis via induction of $\alpha \mathrm{B}$-crystallin and its antiapoptotic function," Neuroscience Letters, vol. 501, no. 3, pp. 132-137, 2011.

[19] J. B. Velotta, N. Kimura, S. H. Chang et al., “ $\alpha$ b-crystallin improves murine cardiac function and attenuates apoptosis in human endothelial cells exposed to ischemia-reperfusion," Annals of Thoracic Surgery, vol. 91, no. 6, pp. 1907-1913, 2011.

[20] W.-F. Hu, L. Gong, Z. Cao et al., " $\alpha \mathrm{A}$ - and $\alpha \mathrm{B}$-crystallins interact with caspase- 3 and bax to guard mouse lens development," Current Molecular Medicine, vol. 12, no. 2, pp. 177-187, 2012.

[21] S. S. Ousman, B. H. Tomooka, J. M. van Noort et al., "Protective and therapeutic role for $\alpha \mathrm{B}$-crystallin in autoimmune demyelination," Nature, vol. 448, no. 7152, pp. 474-479, 2007.

[22] I. J. Benjamin and D. R. McMillan, "Stress (heat shock) proteins molecular chaperones in cardiovascular biology and disease," Circulation Research, vol. 83, no. 2, pp. 117-132, 1998.

[23] R. C. Williams Jr., K. Sugiura, and E. M. Tan, "Antibodies to microtubule-associated protein 2 in patients with neuropsychiatric systemic lupus erythematosus," Arthritis and Rheumatism, vol. 50, no. 4, pp. 1239-1247, 2004.

[24] C. Sánchez, J. Díaz-Nido, and J. Avila, "Phosphorylation of microtubule-associated protein 2 (MAP2) and its relevance for the regulation of the neuronal cytoskeleton function," Progress in Neurobiology, vol. 61, no. 2, pp. 133-168, 2000.

[25] Y. Fujita, E. Ohto, E. Katayama, and Y. Atomi, " $\alpha$ B-Crystallincoated MAP microtubule resists nocodazole and calciuminduced disassembly," Journal of Cell Science, vol. 117, no. 9, pp. 1719-1726, 2004.

[26] Z. Hu, L. Zeng, L. Xie et al., "Morphological alteration of Golgi apparatus and subcellular compartmentalization of TGF- $\beta 1$ 
in Golgi apparatus in gerbils following transient forebrain ischemia," Neurochemical Research, vol. 32, no. 11, pp. 19271931, 2007.

[27] P. R. L. A. van den Ijssela, P. Overkampa, U. Knaufb, M. Gaestelb, and W. W. de Jong, " $\alpha$ A-crystallin confers cellular thermoresistance,” FEBS Letters, vol. 355, no. 1, pp. 54-56, 1994.

[28] P. J. Muchowski and J. L. Wacker, "Modulation of neurodegeneration by molecular chaperones," Nature Reviews Neuroscience, vol. 6, no. 1, pp. 11-22, 2005.

[29] S. Narayanan, B. Kamps, W. C. Boelens, and B. Reif, “ $\alpha$ Bcrystallin competes with Alzheimer's disease $\beta$-amyloid peptide for peptide-peptide interactions and induces oxidation of Abeta-Met35," FEBS Letters, vol. 580, no. 25, pp. 5941-5946, 2006.

[30] J. J. Bajramovic, A. C. Plomp, A. Goes et al., "Presentation of alpha B-crystallin to $\mathrm{T}$ cells in active multiple sclerosis lesions: an early event following inflammatory demyelination," The Journal of Immunology, vol. 164, no. 8, pp. 4359-4366, 2000.

[31] C. S. Piao, S. W. Kim, J. B. Kim, and J. K. Lee, "Co-induction of $\alpha \mathrm{B}$-crystallin and MAPKAPK-2 in astrocytes in the penumbra after transient focal cerebral ischemia," Experimental Brain Research, vol. 163, no. 4, pp. 421-429, 2005.

[32] Z. Hu and T. Li, "HspB5/ $\alpha$ B-crystallin: properties and current progress in neuropathy," Current Neurovascular Research, vol. 5, no. 2, pp. 143-152, 2008.

[33] F. Bennardini, A. Wrzosek, and M. Chiesi, " $\alpha$ B-Crystallin in cardiac tissue: association with actin and desmin filaments," Circulation Research, vol. 71, no. 2, pp. 288-294, 1992.

[34] R. B. Maccioni and V. Cambiazo, "Role of microtubuleassociated proteins in the control of microtubule assembly," Physiological Reviews, vol. 75, no. 4, pp. 835-864, 1995.

[35] D. F. Matesic and R. C. S. Lin, "Microtubule-associated protein 2 as an early indicator of ischemia- induced neurodegeneration in the gerbil forebrain," Journal of Neurochemistry, vol. 63, no. 3, pp. 1012-1020, 1994.

[36] D. A. Dawson and J. M. Hallenbeck, "Acute focal ischemiainduced alterations in MAP2 immunostaining: description of temporal changes and utilization as a marker for volumetric assessment of acute brain injury," Journal of Cerebral Blood Flow and Metabolism, vol. 16, no. 1, pp. 170-174, 1996.

[37] S. M. de Waegh, V. M. Y. Lee, and S. T. Brady, "Local modulation of neurofilament phosphorylation, axonal caliber, and slow axonal transport by myelinating Schwann cells," Cell, vol. 68, no. 3, pp. 451-463, 1992.

[38] S. T. Hsieh, G. J. Kidd, T. O. Crawford et al., "Regional modulation of neurofilament organization by myelination in normal axons," Journal of Neuroscience, vol. 14, no. 11, pp. 6392-6401, 1994.

[39] J. M. Edgar, M. McLaughlin, D. Yool et al., "Oligodendroglial modulation of fast axonal transport in a mouse model of hereditary spastic paraplegia," Journal of Cell Biology, vol. 166, no. 1, pp. 121-131, 2004.

[40] R. Whittaker, M. S. Glassy, N. Gude, M. A. Sussman, R. A. Gottlieb, and C. C. Glembotski, "Kinetics of the translocation and phosphorylation of $\alpha \mathrm{B}$-crystallin in mouse heart mitochondria during ex vivo ischemia," American Journal of Physiology - Heart and Circulatory Physiology, vol. 296, no. 5, pp. H1633-H1642, 2009. 


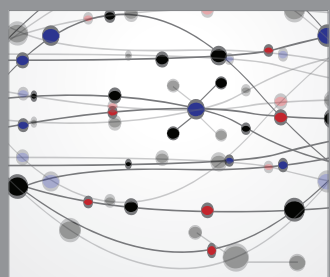

The Scientific World Journal
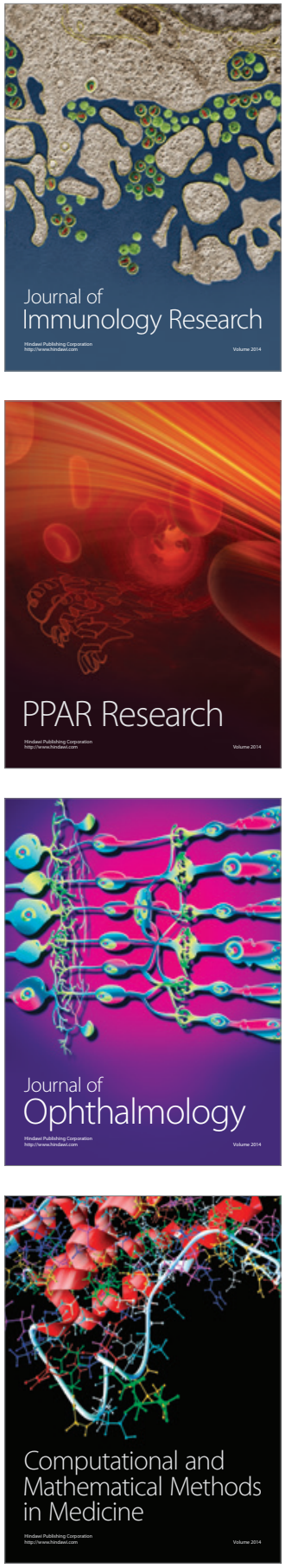

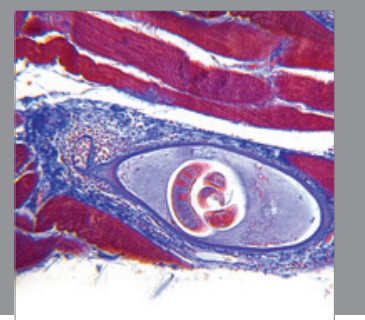

Gastroenterology

Research and Practice
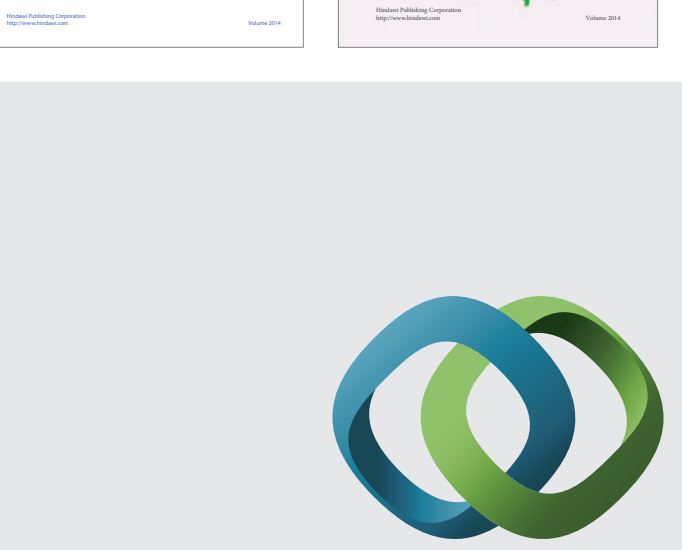

\section{Hindawi}

Submit your manuscripts at

http://www.hindawi.com
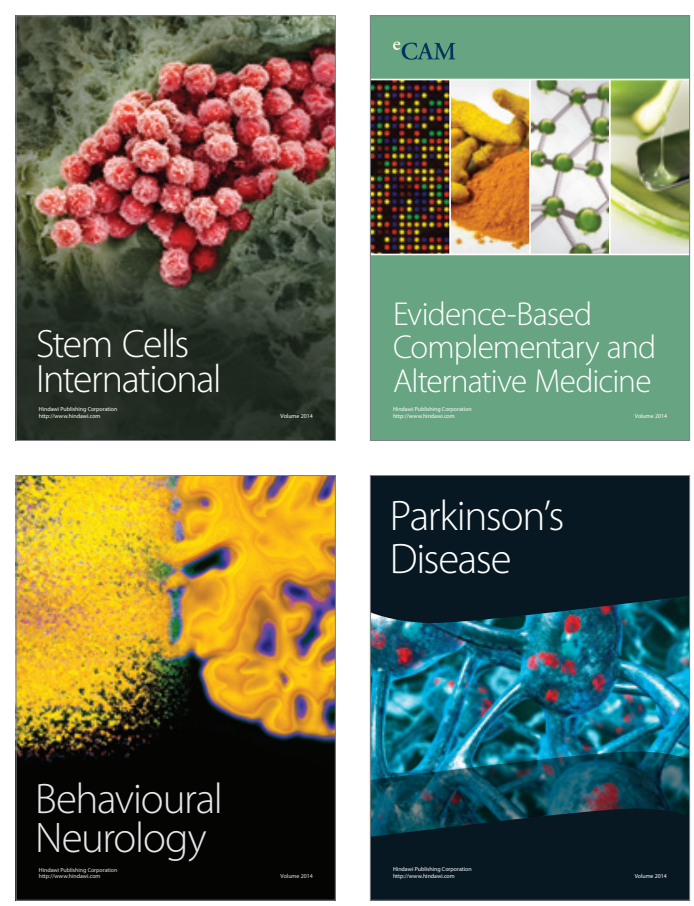

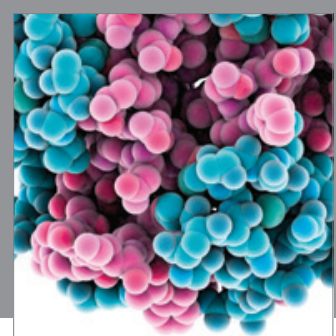

Journal of
Diabetes Research

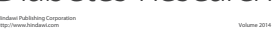

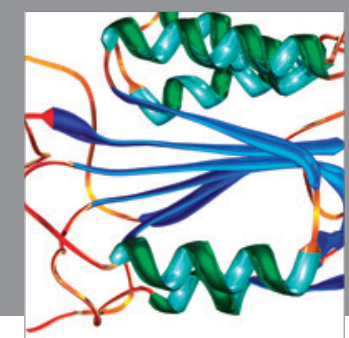

Disease Markers
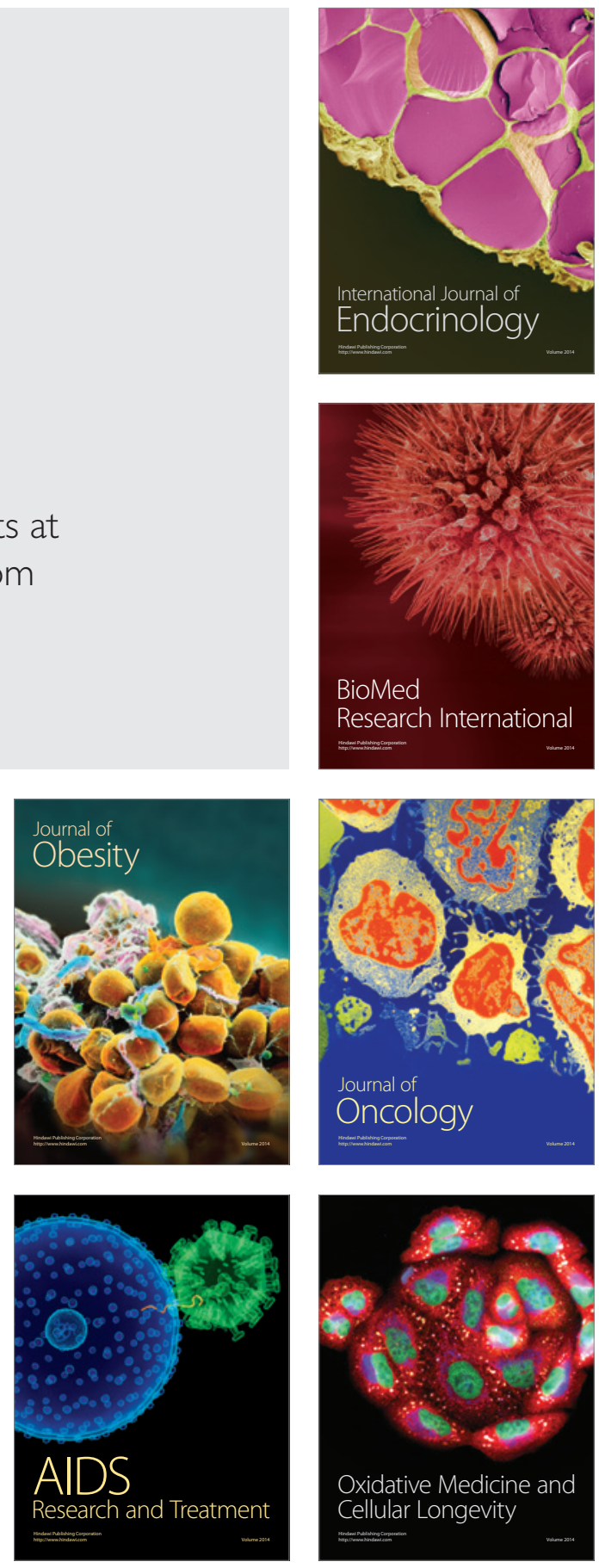